\title{
Contribuições da psicologia e da nutrição para a mudança do comportamento alimentar
}

\author{
Cristineide Leandro França \\ Universidade de Brasilia \\ Marina Biaginni \\ Universidade de Brasilia \\ Ana Paula Levindo Mudesto \\ Ministério da Aeronáutica \\ Elioenai Dornelles Alves \\ Universidade de Brasília
}

\begin{abstract}
Resumo
O estudo avaliou o impacto dos aspectos nutricionais e psicológicos de um grupo psicoeducativo com o foco na mudança do comportamento alimentar. A amostra foi constituída por 11 sujeitos da Universidade de Brasília (UnB), de ambos os sexos, sendo estes, alunos, trabalhadores e seus dependentes na faixa etária de 18 a 70 anos. Foram utilizados a técnica do grupo focal, questionário epidemiológico, de frequência alimentar e aferição do peso e da altura dos participantes. Entre os resultados, confirmou-se a prevalência da depressão e da ansiedade como fatores psicológicos que interferem na mudança de comportamento alimentar e a relevância de uma ação multiprofissional, por meio de grupos psicoeducativos, como forma de modificar o estilo de vida e obter respostas terapêuticas mais eficazes. Além disso, verificou-se em relação ao consumo alimentar uma redução nos grupos dos açúcares, leites e derivados. O peso médio dos participantes apresentou redução significativa.
\end{abstract}

Palavras-chave: nutrição; psicologia; enfermagem; comportamento alimentar; grupos psicoeducativos.

\begin{abstract}
Contributions of psychology and nutrition to the change of eating behavior. The study evaluated the impact of a psychoeducational group focused on change in eating behavior over nutritional and psychological aspects of the participants. The sample consisted of 11 subjects of both genders, among which were students from the University of Brasília, as well as employees and their legal dependents, all aged from 18 to 70 years. The techniques of the focus group, epidemiological questionnaire, food frequency and the measurement of weight and height were all applied to the study. The results confirmed the prevalence of depression and anxiety as psychological factors that influence the change in eating behavior and the relevance of a multiprofessional action by psychoeducational groups as a way of modifying the patient's lifestyle and getting more effective therapeutic responses. Furthermore, a reduction on the consumption of food pertaining to the groups of sugar and of milk and its derivatives was also verified. Finally, the average weight of participants decreased during the experiment.
\end{abstract}

Keywords: psychology; nutrition; nursing; eating behavior; psychoeducational group

É preocupante o aumento da obesidade e sobrepeso da população mundial neste século. $\mathrm{O}$ excessivo e anormal acúmulo de gordura é considerado atualmente pela Organização Mundial de Saúde (OMS) um problema de saúde pública, medidos pelo índice de massa corporal (IMC) de $30 \mathrm{~kg} / \mathrm{m}^{2}$ ou mais para obeso e igual ou superior a $25 \mathrm{~kg} /$ $\mathrm{m}^{2}$ para sobrepeso. Essas doenças ocasionam grande risco à saúde da população para enfermidades como diabetes, doenças cardiovasculares, câncer (ABESO, 1998; OMS, 2008) e manifestações dermatológicas (Boza, Rech, Sachett, Menegon, \& Cestari, 2010). Além do dano físico, estão relacionadas à incidência de distúrbios psicológicos como ansiedade, depressão, transtornos alimentares, baixa autoestima e imagem corporal distorcida (INCA, 2004; Lawrence \& Kopelman, 2004). Fatores psicológicos que influenciam no comportamento alimentar, como depressão, ansiedade e sentimentos inespecíficos de tensão são identificados como ativadores da compulsão alimentar. Indivíduos com esse padrão alimentar apresentam, além da ansiedade e depressão, mais frequentemente sentimentos de autodesprezo, repulsa a forma corporal, preocupação somática, estresse e prejuízo em suas relações interpessoais (Perez \& Romano, 2004).

$\mathrm{Na}$ esfera mundial, conforme dados da OMS (2008), a obesidade mais que dobrou desde a década de 1980. Em 2008, 
1,5 bilhão de adultos com 20 anos ou mais estavam acima do peso. Desses, mais de 200 milhões eram homens e quase 300 milhões eram mulheres. Dados desta pesquisam revelam que $65 \%$ da população mundial vive em países onde o sobrepeso e a obesidade matam mais pessoas do que o baixo peso. Além disso, obesidade e sobrepeso são consideradas não mais como um problema apenas dos países de renda elevada, pois suas taxas têm aumentado de forma signiticativa nos países de baixa e média renda, especialmente em áreas urbanas, atingindo índices avaliados como de doenças epidêmicas.

No Brasil, o excesso de peso tem tido grande prevalência nas últimas décadas. Em 2002, foi estimada em 40\% entre as mulheres e $41 \%$ entre os homens (IBGE, 2004). Ainda segundo dados do IBGE, em pesquisa feita em 2008 e 2009, o excesso de peso afetou cerca de metade dos homens e das mulheres, excedendo em 28 vezes a frequência do déficit de peso no caso masculino e em 13 vezes no feminino e atingiram duas a três vezes mais os homens de maior renda, além de se destacarem nas regiões Sudeste, Sul e Centro-Oeste e nos domicílios urbanos. Nas mulheres, as duas condições se destacaram no Sul do país e nas classes intermediárias de renda.

As razões para esse aumento significativo podem estar associadas à inatividade física da população e a uma maior ingestão de alimentos altamente energéticos como gordura, sal, açúcares e deficitários em vitaminas, minerais e outros micronutrientes. Medidas preventivas são necessárias para o controle do sobrepeso e obesidade. Entre estas estão ações que estimulem modificações efetivas no estilo de vida, nos padrões alimentares e de atividade física (Katz, Feigenbaum, Pasternary, \& Vinker, 2005; Perez \& Romano, 2004) e investimentos em mudanças ambientais, familiares e sociais. É recomendado, ainda, pela OMS, apoio político às ações em áreas da saúde, agricultura, transportes, planejamento urbano, meio ambiente, processamento de alimentos, distribuição, marketing e educação.

Como estratégia global de prevenção e controle das doenças não transmissiveis, a OMS desenvolveu o Plano de Ação de 2008-2013 com o objetivo de ajudar milhões de pessoas que já estão afetadas a lidar com essas doenças e prevenir complicações secundárias. Este plano de ação destina-se a construir políticas e alternativas para o controle do tabaco e estratégia global sobre alimentação, atividade física e saúde.

Como parte desta estratégia, o Ministério da Saúde lançou em 2008 o Guia alimentar para a população brasileira, que traz diretrizes e recomendações ditadas pela OMS e corrobora com as seguintes orientações específicas sobre dieta constantes no documento final da Estratégia Global de Promoção da Alimentação, Atividade Física e Saúde: manter o equilíbrio energético e o peso saudável; limitar a ingestão energética procedente de gorduras; substituir as gorduras saturadas por insaturadas e eliminar as gorduras trans (hidrogenadas); aumentar o consumo de frutas, legumes e verduras, cereais integrais e leguminosas (feijões); limitar a ingestão de açúcar livre; limitar a ingestão de sal (sódio) de toda procedência e consumir sal iodado.

Apesar de informações sobre os prejuízos das doenças crônicas não-transmissíveis estarem amplamente divulgadas nos últimos anos, a prevenção e o tratamento destas enfermidades por meio da mudança no estilo de vida, possuem uma adesão de apenas 25\% dos pacientes (DiMatteo, 1994). Um dos motivos para a baixa adesão às mudanças no estilo de vida relacionase ao uso adequado do conhecimento, pois este só serve de instrumento para a mudança se houver no indivíduo o desejo de mudar (Chapman, Ham, Liesen, \& Winter, 1995). Sendo assim, causas da baixa adesão podem estar relacionadas à inadequação de estratégias e intervenções utilizadas no tratamento dessas doenças.

Entre os tratamentos não farmacológicos para problemas de saúde estão as intervenções em grupo interdisciplinar apontada pela literatura (Cezaretto, 2010; Murta, 2005; Santos, 2010; Tremblay, Sheeran, \& Aranda, 2008) como ações benéficas para a mudanças de hábitos, sintomas e construção de competências relacionadas à saúde e qualidade de vida da população. Além disso, segundo Murta (2008), ações preventivas e de tratamento em grupo são de baixo custo, atingem uma quantidade maior de pessoas do que intervenções individuais proporcionam a reconstrução de crenças e estimula a motivação e a autoeficácia dos participantes.

Nesse sentido, as intervenções grupais estão em consonância com as diretrizes da saúde pública e princípios básicos de universalidade, integralidade da atenção e controle social. Tais ações podem auxiliar na democracia ao acesso à saúde, estimular a inclusão da comunidade nos cuidados e atenção integral conforme suas necessidades específicas, crenças, costumes e gestão dos serviços (Rasera \& Rocha, 2010).

Sharovsky, Perez, Romano e Lopes (2004) verificaram que a intervenção grupal melhora o funcionamento psíquico do paciente. Mesmo nos casos em que o tratamento de escolha para obesidade foi prioritariamente farmacológico, a inserção de grupos voltados para mudança do estilo de vida impôs maior emagrecimento dos pacientes.

Um estudo longitudinal realizado por Cezarreto (2010), com indivíduos pré-diabéticos ou portadores de síndrome metabólica, tinha como objetivo verificar a melhoria na qualidade de vida desses pacientes após alguns serem submetidos a uma intervenção interdisciplinar em grupo (grupo I) e outros a uma intervenção tradicional (grupo II). O grupo I foi submetido a consultas médicas tradicionais com endocrinologista a cada três meses. O grupo II foi submetido a consultas trimestrais com o endocrinologista, a orientações dietéticas individuais com uma nutricionista e à participação em grupo psicoeducativo interdisciplinar, com 13 sessões. A intervenção psicoeducativa utilizou material informativo, dinâmicas de grupo e instrumentos psicométricos. Constatou-se nesse estudo que uma intervenção interdisciplinar em grupo trouxe benefícios metabólicos e extrametabólicos a indivíduos de risco para diabetes mellitus tipo 2, reduziu sintomas depressivos e de compulsão alimentar e melhorou a qualidade de vida dos participantes.

De modo semelhante, Santos (2010) realizou estudo quase-experimental, com objetivo de avaliar os efeitos de um programa interdisciplinar em grupo para a mudança no comportamento alimentar. A intervenção foi coordenada por uma psicóloga e nutricionista e consistia de oito sessões com duas horas de duração cada, com o uso de técnicas comportamentais cognitivas. A Terapia Comportamental Cognitiva - TCC é uma 
psicoterapia breve, estruturada, orientada para o presente, direcionada a resolver problemas atuais e modificar pensamentos e comportamentos disfuncionais (Rangé, 2001), portanto, uma ferramenta adequada para intervenções grupais com foco na mudança de comportamento alimentar. Os resultados do estudo de Santos (2010) apontam a efetividade de um trabalho interdisciplinar para pessoas obesas e com sobrepeso, uma melhora nos sintomas de compulsão alimentar periódica, na qualidade da alimentação e o aumento do conhecimento sobre o próprio hábito alimentar.

Nesse contexto, considerando os altos índices de doenças relacionadas à obesidade e sobrepeso no Brasil e no mundo, torna-se necessário introduzir práticas de saúde baseadas em modelos eficazes. As intervenções em grupo são alternativas que podem estimular e motivar o indivíduo à mudança de comportamento alimentar para uma vida mais saudável. Assim, o presente estudo visa avaliar o impacto imediato dos aspectos nutricionais e psicológicos de um grupo psicoeducativo com o foco na mudança do comportamento alimentar. Para tanto, foram definidos os seguintes objetivos: (a) avaliar modificações no peso e no consumo alimentar dos participantes após o grupo psicoeducativo; (b) identificar fatores psicológicos que interferem na mudança do comportamento alimentar e (c) analisar a eficácia do grupo psicoeducativo, em uma ação multiprofissional, para a promoção da mudança no comportamento alimentar.

\section{Material e Método}

\section{Participantes}

A amostra foi constituída por 2 homens e 11 mulheres, com idade entre 22 e 62 anos, escolaridade que variou de ensino básico incompleto a nível superior, sendo alunos, trabalhadores e seus dependentes, todos da Universidade de Brasília (UnB). Dos 13 sujeitos, dois, do sexo feminino, não participaram até a sessão final, ficando a amostra com 11 sujeitos. Estes dados estão apresentados mais detalhadamente na Tabela 1. Os participantes selecionados foram encaminhados pela equipe multiprofissional do setor de qualidade de vida, composta por médicos, psicólogos, enfermeiros, assistente social e nutricionistas. Em seguida, foram realizadas consultas individuais com a nutricionista e a psicóloga responsáveis pelo grupo psicoeducativo, com o objetivo de verificar se os sujeitos tinham os pré-requisitos necessários para inserção no grupo.

Tabela 1

Características sociodemográficas e epidemiológicas dos sujeitos.

\begin{tabular}{|c|c|c|c|}
\hline Variáveis & Níveis & $\begin{array}{l}\text { Frequência } \\
\text { absoluta } \\
(n=11)\end{array}$ & $\begin{array}{l}\text { Frequência } \\
\text { relativa }(\%)\end{array}$ \\
\hline \multirow[t]{2}{*}{ Gênero } & Feminino & 9 & 81,8 \\
\hline & Masculino & 2 & 18,2 \\
\hline \multirow[t]{5}{*}{ Idade } & Até 29 anos & 2 & 18,2 \\
\hline & 30 a 39 anos & 4 & 16,4 \\
\hline & 40 a 49 anos & 2 & 18,2 \\
\hline & 50 a 59 anos & 2 & 18,2 \\
\hline & 60 anos ou mais & 1 & 9,1 \\
\hline \multirow[t]{3}{*}{ Escolaridade } & Ensino básico ou menos & 4 & 36,4 \\
\hline & Ensino médio & 5 & 45,5 \\
\hline & Ensino superior & 2 & 18,2 \\
\hline \multirow[t]{5}{*}{ Problemas de saúde } & Hipertensão arterial sistêmica & 5 & 45,4 \\
\hline & Diabetes mellitus & 2 & 18,2 \\
\hline & Dor na coluna, membros inferiores ou articulações & 7 & 63,6 \\
\hline & Varizes & 3 & 27,3 \\
\hline & Outros & 2 & 18,2 \\
\hline \multirow[t]{2}{*}{ Hábito de fumar } & Sim & 1 & 9,1 \\
\hline & Não & 10 & 90,9 \\
\hline \multirow[t]{2}{*}{ Prática de atividade física } & $\operatorname{Sim}$ & 7 & 63,6 \\
\hline & Não & 4 & 36,4 \\
\hline
\end{tabular}

Os critérios para inclusão no estudo foram: (a) apresentar alguma doença crônica não-transmissível cujo tratamento inclui a dietoterapia, como sobrepeso, obesidade, hipertensão arterial, diabetes melito, dislipidemias e outras; (b) ter alguma queixa psicológica que influenciasse no comportamento alimentar; e (c) não possuir bulimia ou anorexia nervosa.

\section{Instrumentos}

Como instrumento para caracterizar o grupo estudado, foi elaborado pelos pesquisadores um questionário que investigava questões como dados pessoais, estado de saúde, história familiar de doenças, hábito de fumar e prática de atividade física. Este questionário foi aplicado na primeira sessão do grupo. 
O consumo alimentar habitual dos participantes foi avaliado por meio de um Questionário de Frequência Alimentar (QFA) previamente validado por Furlan-Viebig e Pastor-Valero (2004), aplicado na primeira e na última sessão do grupo. Este questionário avaliou a frequência de consumo de diversos alimentos, classificados nos seguintes grupos: leite e derivados, carnes, vegetais, frutas, cereais, gorduras, doces, bebidas e preparações.

Foi solicitado aos participantes do grupo o preenchimento do QFA, com prévia explicação oral sobre como preenchê-lo, sendo destinado um momento da primeira e da última sessão para isso. O participante deveria assinalar a coluna que correspondesse ao seu consumo de cada um dos alimentos, classificado em: nunca ou menos de 1 vez ao mês, 2 a 3 vezes por mês, 1 vez por semana, 2 a 4 vezes por semana, 5 a 7 vezes por semana, 1 vez por dia, 2 a 3 vezes por dia, 4 a 6 vezes por dia, mais de 6 vezes por dia. Para a avaliação do QFA, foi utilizada a metodologia proposta por Monteiro, Riether, Trapp e Burini (2004), que usa o cômputo geral da frequência de consumo transformando todas as frequências em porções diárias. Ou seja, um consumo diário é escrito como $1 / 1$ ou 1,0 ; um consumo semanal equivale a $1 / 7$ ou 0,14 ; um consumo mensal equivale a $1 / 30$ ou 0,03 ; e assim sucessivamente.

Os dados sobre consumo alimentar obtidos através do QFA foram em seguida comparados com as diretrizes do Guia Alimentar para a População Brasileira (Ministério da Saúde, 2008), com o objetivo de avaliar se os padrões de consumo do grupo condiziam com uma alimentação saudável.

Foi aferido, também na primeira e na última sessão, o peso e a estatura dos participantes. O peso foi aferido com balança hospitalar da marca Filizola com capacidade de até $150 \mathrm{~kg}$ e precisão de $100 \mathrm{~g}$, e os participantes estavam sem sapatos, agasalho ou objetos nos bolsos (Fonseca, Faerstein, Chor, \& Lopes, 2004). A aferição da estatura foi feita com o estadiômetro embutido na mesma balança com os participantes em posição ereta, com os braços estendidos para baixo e os pés unidos (Fonseca, Faerstein, Chor, \& Lopes, 2004). Depois de obtidos o peso e a estatura dos participantes foi determinado o índice de massa corporal calculado, dividindo-se o peso em quilos pelo quadrado da estatura em centímetros (Anjos, 1992).

Para avaliação dos fatores psicológicos propostos no estudo, utilizou-se a técnica de grupo focal, em dois momentos: segunda e última sessão da intervenção psicoeducativa, com duração de 60 minutos cada. Optou-se por este método de pesquisa qualitativa por ser útil no entendimento de como se formam as diferentes percepções e atitudes acerca de um fato, prática, produto ou serviços (Krueger, 1988).

Nessa perspectiva, a psicóloga atuou como moderadora, explicando aos participantes os objetivos do encontro, a garantia do sigilo, a forma de registro dos dados e esclarecendo sobre a importância de todas as opiniões (Gatti, 2005). A nutricionista exerceu o papel de observador, auxiliando na anotação das falas. Utilizaram-se como material dois gravadores digitais portáteis e cadeiras avulsas em círculo. No primeiro grupo focal buscou-se investigar as percepções sobre fatores psicológicos que interferem na mudança do comportamento alimentar e conhecer as estratégias de intervenção utilizadas para amenizar tais sintomas psicológicos.

O segundo grupo focal investigou a eficácia do grupo psicoeducativo na mudança do comportamento alimentar. $\mathrm{O}$ material obtido nos grupos focais foi gravado, posteriormente transcrito e, para tratamento dos dados, utilizou-se a técnica de análise categorial temática de Bardin (2007). Dessa forma, buscou-se analisar as falas dos participantes conforme as fases da análise de conteúdo: a pré-exploração ou leitura flutuante do material; a seleção das unidades de análise/significado e o processo de categorização.

\section{Procedimentos}

Entre a realização dos dois grupos focais, que aconteceu no prazo de três meses, ocorreu a intervenção grupal psicoeducativa. Essa intervenção consistiu em motivar os participantes a investirem em estratégias de autocuidado, informar e esclarecer sobre consequências física e psicológica do excesso de peso e dar orientações nutricionais que auxiliassem os participantes a escolher os alimentos mais saudáveis nas diversas situações do quotidiano.

A intervenção foi organizada e coordenada por uma nutricionista e psicóloga que trabalham na Coordenação de Qualidade de vida- CQV no Hospital Universitário de Brasília HUB e ocorreu na sala de grupo da CQV/HUB, em 13 encontros semanais, com duração de 120 minutos cada. Os temas e atividades principais das sessões estão apresentados na Tabela 2.

Nas sessões foram utilizadas técnicas de cunho psicoeducativo com base na terapia cognitiva comportamentalTCC. Os temas foram trabalhados em forma de dinâmicas de grupo, palestras e atividades práticas com a presença da psicóloga e da nutricionista em todas as sessões. A quinta e a décima segunda sessão contaram com a presença e orientações de profissionais de saúde como educador físico e enfermeiro.

Os cuidados com aspectos éticos da pesquisa foram apresentados no primeiro encontro por meio de contrato verbal com os participantes que foram informados sobre objetivos e procedimentos e convidados a assinarem o Termo de Consentimento Livre e Esclarecido. A realização da pesquisa recebeu parecer favorável da Comissão de Ética da Faculdade de Ciências da Saúde - FS /UnB (registro do projeto 121/09) e anuência do HUB.

\section{Resultados}

Os resultados psicológicos apresentados neste estudo contemplaram 03 categorias temáticas que foram agrupadas conforme as questões que nortearam os dois grupos focais: (1) Fatores psicológicos; (2) Estratégias de intervenção; e (3) Eficácia do grupo psicoeducativo.

A análise dos dados da categoria (1) fatores psicológicos aborda a percepção do grupo sobre a interferência de aspectos psicológicos na manutenção de hábitos alimentares adequados. Essa categoria possibilitou identificar sentimentos como: tristeza, raiva, depressão, ansiedade, dificuldades no relacionamento familiar, baixa autoestima, avaliação negativa da imagem corporal, motivação e alegria. Constataram-se, nessa amostra, que a depressão e a ansiedade foram as respostas predominantes. 
Tabela 2

Descrição dos temas, atividades principais e objetivos da intervenção.

\begin{tabular}{|c|c|c|c|}
\hline Sessão & Tema & Atividades Principais & Objetivos \\
\hline $1^{\mathrm{a}}$ & Apresentação do grupo & $\begin{array}{l}\text { TCLE, programação do grupo, diário } \\
\text { alimentar, avaliação inicial }\end{array}$ & $\begin{array}{l}\text { Cuidados éticos, } \\
\text { objetivos do grupo }\end{array}$ \\
\hline $2^{\mathrm{a}}$ & $\begin{array}{l}\text { Princípios da alimentação } \\
\text { saudável }\end{array}$ & $\begin{array}{l}\text { Grupo Focal, brainstorm, exposição } \\
\text { dialogada }\end{array}$ & $\begin{array}{l}\text { Iniciar o diálogo sobre alimentação } \\
\text { saudável }\end{array}$ \\
\hline $3^{\mathrm{a}}$ & Peso saudável & $\begin{array}{l}\text { Cartão de enfrentamento, exposição } \\
\text { dialogada }\end{array}$ & $\begin{array}{l}\text { Fortalecer a motivação, enfraquecer } \\
\text { pensamentos sabotadores, } \\
\text { introduzir o conceito de peso } \\
\text { saudável }\end{array}$ \\
\hline $4^{\mathrm{a}}$ & $\begin{array}{l}\text { Grupo de alimentos e suas } \\
\text { funções }\end{array}$ & $\begin{array}{l}\text { Exposição de recipientes contendo } \\
\text { óleo, açúcar e sal contidos nos } \\
\text { alimentos; atividade de colagem; } \\
\text { exposição dialogada }\end{array}$ & $\begin{array}{l}\text { Esclarecer a importância dos } \\
\text { diferentes grupos de alimentos }\end{array}$ \\
\hline $5^{\mathrm{a}}$ & Atividade física & $\begin{array}{l}\text { Prática de exercícios físicos no Centro } \\
\text { Olímpico com um profissional de } \\
\text { educação física }\end{array}$ & $\begin{array}{l}\text { Incentivar a prática de atividade } \\
\text { física }\end{array}$ \\
\hline $6^{\mathrm{a}}$ & Dietas da moda & $\begin{array}{l}\text { Discussão sobre dietas propostas por } \\
\text { revistas e páginas da internet, técnica } \\
\text { de relaxamento }\end{array}$ & $\begin{array}{l}\text { Discutir os prós e contras das dietas } \\
\text { não baseadas na alimentação } \\
\text { saudável, combater a ansiedade }\end{array}$ \\
\hline $7^{\mathrm{a}}$ & Rotulagem de alimentos & $\begin{array}{l}\text { Observação e comparação de rótulos } \\
\text { de alimentos }\end{array}$ & $\begin{array}{l}\text { Ensinar como utilizar o rótulo na } \\
\text { escolha dos alimentos }\end{array}$ \\
\hline $8^{\mathrm{a}}$ & Refeições saudáveis & $\begin{array}{l}\text { Vivência "construção de um prato } \\
\text { saudável", automonitoramento }\end{array}$ & $\begin{array}{l}\text { Motivar a mudança de hábitos } \\
\text { alimentares e tomada de decisão }\end{array}$ \\
\hline $9^{\mathrm{a}}$ & $\begin{array}{l}\text { Mitos e verdades sobre o } \\
\text { emagrecimento }\end{array}$ & $\begin{array}{l}\text { Dinâmica "escravos de Jó", discussão } \\
\text { de mitos do emagrecimento }\end{array}$ & $\begin{array}{l}\text { Treinar o elogio, esclarecer mitos } \\
\text { em relação ao emagrecimento }\end{array}$ \\
\hline $10^{\mathrm{a}}$ & $\begin{array}{l}\text { Uso de medicação para } \\
\text { emagrecer }\end{array}$ & $\begin{array}{l}\text { Apresentação do filme "Requiem para } \\
\text { um sonho" }\end{array}$ & $\begin{array}{l}\text { Conhecer prós e contras da } \\
\text { medicação para emagrecer }\end{array}$ \\
\hline $11^{\mathrm{a}}$ & Hábitos saudáveis & $\begin{array}{l}\text { Vivência "construção de um prato } \\
\text { saudável", automonitoramento }\end{array}$ & $\begin{array}{l}\text { Motivar a mudança de hábitos } \\
\text { alimentares e tomada de decisão }\end{array}$ \\
\hline $12^{\mathrm{a}}$ & $\begin{array}{l}\text { Uso de plantas medicinais } \\
\text { para a saúde }\end{array}$ & $\begin{array}{l}\text { Visita ao herbário do Centro de } \\
\text { Medicina Alternativa - CEMA /DF }\end{array}$ & $\begin{array}{l}\text { Conhecer riscos e benefícios das } \\
\text { plantas medicinais }\end{array}$ \\
\hline $13^{\mathrm{a}}$ & $\begin{array}{l}\text { Encerramento, } \\
\text { encaminhamentos e } \\
\text { avaliações }\end{array}$ & $\begin{array}{l}\text { Grupo focal, avaliação final e lanche } \\
\text { saudável }\end{array}$ & Avaliar o grupo, fornecer feedback \\
\hline
\end{tabular}

Algumas falas são representativas dessa categoria:

...Eu acho que além da tristeza que a pessoa passa, tem os problemas na família. Pode ser a questão de ter problemas com marido, filhos, falta de dinheiro, amor, carinho, a bebida... Se eu estou fazendo a dieta, eu paro o que estou fazendo e passo a comer tudo. Ai eu penso: meu deus, por que eu fiz isso?

... Isso também se passa comigo. Eu tenho depressão... Às vezes fico em casa trabalhando, fazendo a dieta, o meu marido chega do trabalho, não conversa nada, toma banho e vai dormir. Não me dá atenção e eu fico triste, com raiva, choro e aí como tudo que vejo pela frente...

Em seguida, avaliou-se a categoria (2), estratégias de intervenção, que teve como intuito investigar como os participantes se comportam diante de sintomas psicológicos que influenciam negativamente na mudança dos hábitos alimentares. Os princípios da terapia cognitiva comportamental - TCC auxiliaram na construção dessa categoria. A técnica da distração e a modificação de crenças disfuncionais são algumas estratégias da TCC que foram utilizadas pelo grupo. A distração consiste em deslocar a atenção do mundo interno (pensamentos, sensações corporais) para o mundo externo (Knapp \& Baldisserotto, 2001). Ao modificar sua forma de pensar, os participantes aprendem como mudar seus pensamentos imprecisos e disfuncionais para que se sintam melhores emocionalmente e para que se comportem de maneira mais produtiva na busca das suas metas (Beck, 2009).

Os participantes relataram aspectos que deram origem, nessa categoria, aos seguintes temas: ir à igreja, realizar leituras, trabalhar, usar o computador, meditar, realizar atividades físicas, ir ao shopping e fazer trabalhos manuais. A realização de atividades físicas foi a resposta com maior frequência. Para eles, essas estratégias ajudam a controlar os problemas psicológicos e a lidar com os impulsos com relação à fissura por comida. Alguns depoimentos são representativos e ilustram a opinião do grupo sobre essa temática:

“... fazer cartazes nos trabalhos da igreja e ficar no 
computador me ajuda a tirar o foco da comida e manter minha dieta". "... Faço crochê, procuro trabalhar nos concursos, vou para igreja. Assim me distraio e evito pensamentos ruins". “... Eu gosto de passear no shopping e gosto de caminhar. Isso preenche minha cabeça".

Quando investigados sobre (3) eficácia do grupo psicoeducativo na mudança do comportamento alimentar, os participantes relataram que participar do grupo proporcionou melhora da autoestima, mudança de hábitos, realização de atividade física com maior frequência, compartilhar problemas com os outros, mudança de pensamento, valorização dos alimentos, perda de peso, melhora de indicadores bioquímicos como colesterol, triglicerídeos e glicemia e escolha alimentares saudáveis. A melhora da autoestima foi a resposta com maior ocorrência enfatizada pelos participantes. Algumas verbalizações são exemplos dessa categoria temática, a seguir: "Aprendi a valorizar os alimentos, melhorei minha autoestima. Estou fazendo bicicleta... Agora faço refeições com outro prazer". "Melhorou minha autoestima. Vi que existem outras pessoas como eu. Comecei a nadar e estou pedalando. Não ligo de não ter perdido peso". "Gostei muito do grupo. Me ajudou muito. Aprendi técnicas de distração". O grupo foi importante para ter uma orientação mais aprofundada. Tenho que mudar, mas já mudei muito. Reduzi refrigerantes e comidas gordurosas. Meus exames também melhoraram.

Os dados referentes ao consumo alimentar e ao índice de massa corporal foram analisados com o auxílio do programa SPSS Statistics versão 18, sendo realizado o teste T para amostras pareadas. Dos 11 participantes do grupo, 10 tiveram o peso e a estatura aferidos e 5 preencheram os questionários de frequência alimentar, sendo os dados perdidos desconsiderados em cada uma das análises estatísticas.
A média do IMC dos participantes na primeira e na última sessão do grupo foi significantemente diferente, apresentando uma redução (Tabela 3).

Em relação ao consumo alimentar dos participantes, não foi verificada diferença significativa em nenhum dos grupos de alimentos entre a primeira e a última sessão do grupo. Porém, os valores médio, mínimo e máximo de cada grupo de alimentos, principalmente dos grupos de leite e derivados, doces, bebidas adoçadas e preparações (Tabela 4), aproximaram-se dos valores preconizados pelo Guia alimentar para a população brasileira (Ministério da Saúde, 2008) ao final do grupo psicoeducativo.

\section{Discussão}

A análise dos fatores psicológicos aborda a percepção do grupo sobre a interferência de aspectos emocionais na manutenção de hábitos alimentares adequados. Sentimentos avaliados pelo grupo como negativos possibilitaram uma adesão menor à dieta e à prática de hábitos saudáveis. Ao sentirem tristeza, raiva, depressão, ansiedade, dificuldades no relacionamento familiar, baixa autoestima e avaliação negativa da imagem corporal, os participantes relataram que se desestimulavam a continuar a fazer dieta, praticar exercícios físicos e cuidar de si mesmas.

Por outro lado, sentimentos positivos como motivação e alegria foram avaliados nessa amostra, como força propulsora à mudança do comportamento alimentar e à prática de hábitos saudáveis. Assim, motivação é fator primordial para a adesão às mudanças no estilo de vida Segundo Chapman et. al., (1995), o conhecimento só serve de instrumento para a mudança se houver no indivíduo o desejo de mudar.

Os dados ainda evidenciaram que os participantes utilizam estratégias de intervenção como forma de lidar com

Tabela 3

Média do IMC dos participantes no início e no final do grupo psicoeducativo $(n=10)$.

\begin{tabular}{lccccc}
\hline & \multicolumn{2}{c}{ Início do grupo } & \multicolumn{2}{c}{ Final do grupo } & Valor $p$ \\
\hline & Média & Desvio padrão & Média & Desvio padrão & \\
\cline { 2 - 6 } IMC $\left(\mathrm{kg} / \mathrm{m}^{2}\right)$ & 32,96 & 4,96 & 32,54 & 4,89 & $0,036^{*}$ \\
\hline
\end{tabular}

*Valor significativo (ponto de corte: $p<0,05$ )

Tabela 4

Média de consumo diário dos grupos de alimentos no início e no final do grupo psicoeducativo $(n=5)$.

\begin{tabular}{lccccccc}
\hline \multicolumn{1}{c}{$\begin{array}{c}\text { Grupos de } \\
\text { alimentos }\end{array}$} & \multicolumn{3}{c}{ Início do grupo } & & Final do grupo & Valor $p^{*}$ \\
\hline & Média & Mínimo & Máximo & Média & Mínimo & Máximo \\
\cline { 2 - 7 } Leite e derivados & 4,48 & 1 & 10 & 2,23 & 1 & 3 & 0,215 \\
Carnes & 1,05 & 0 & 2 & 1,21 & 0 & 2 & 0,495 \\
Vegetais & 3,51 & 2 & 4 & 4,33 & 2 & 8 & 0,537 \\
Frutas & 5,23 & 3 & 8 & 5,47 & 3 & 7 & 0,771 \\
Pães, cereais e & 6,19 & 4 & 9 & 5,33 & 3 & 7 & 0,594 \\
tubérculos & 1,27 & 0 & 4 & 1,48 & 1 & 2 & 0,803 \\
Gorduras & 5,87 & 2 & 19 & 1,95 & 1 & 4 & 0,219 \\
Doces & 1,73 & 0 & 4 & 0,95 & 0 & 2 & 0,227 \\
Bebidas adoçadas & 0,83 & 0 & 2 & 0,35 & 0 & 1 & 0,155 \\
Preparações & & & & & & & 7 \\
\hline
\end{tabular}

*Valores não significativos (ponto de corte: $p<0,05$ ) 
esses sintomas psicológicos que interferem na mudança do comportamento alimentar. Ações como ir à igreja, realizar leituras, trabalhar, usar o computador, meditar, realizar atividades físicas, ir ao shopping e fazer trabalhos manuais foram relatadas. A estratégia predominante foi a realização de atividades físicas. Logo, esta forma eficiente de intervenção está em consonância com alguns estudos (Monteiro et al., 2004; Perez \& Romano, 2004), os quais apontam que a prática regular de exercícios físicos traz amplos benefícios à saúde física e mental, entre eles a prevenção e o controle da obesidade.

Os resultados também permitiram avaliar a eficácia do grupo psicoeducativo em uma ação multiprofissional, para a promoção da mudança no comportamento alimentar. A intervenção em grupo mostrou-se efetiva na promoção de hábitos alimentares saudáveis e na modificação do comportamento alimentar, fato também descrito nos estudos de Cezarreto (2010); Santos (2010) e Sharovsky et al. (2004), que destacam a relevância de uma intervenção multiprofissional para a saúde física e psíquica do paciente, auxiliando no emagrecimento. Assim, essa ação conjunta, entre profissionais de saúde, aliada à terapia cognitiva comportamental - TCC propicia benefícios.

De forma semelhante, alguns programas de educação nutricional têm associado às informações sobre hábitos alimentares às teorias motivacionais e treinamento de autocontrole a fim de obter um resultado melhor (Assis \& Nahas, 1999; Cade et al., 2009; Souza et al., 2005). Dessa forma, destaca-se que psicólogos e nutricionistas podem e devem trabalhar em conjunto com outros especialistas da Saúde e da Educação ao serem definidos programas de educação para a saúde (Viana, 2002).

Em relação ao perfil epidemiológico desse grupo, verificouse que as mulheres mostraram mais atenção à alimentação saudável e perda de peso. Além disso, o fato de apenas um participante ter relatado não fazer atividade física regularmente mostra que o grupo em geral já tinha desde o início uma preocupação com a saúde e perda de peso.

$\mathrm{O}$ grupo mostrou um resultado positivo em relação à antropometria, com uma redução significativa na média do IMC dos participantes e modificações no padrão alimentar do grupo, com modificação no consumo de leite e derivados, doces, bebidas adoçadas e preparações. Com relação ao grupo de leite e derivados, observou-se inicialmente uma disparidade no consumo. Embora a média inicial de porções tenha sido próxima ao que recomenda o Guia Alimentar para a População Brasileira (três porções diárias), o consumo entre os participantes apresentou variação importante: enquanto alguns não atingiram a recomendação do guia, ingerindo uma porção diária, outros excederam, em muito, o que foi preconizado nas orientações, ingerindo cerca de dez porções diárias. Ao final da intervenção psicoeducativa, a ingestão de leite e derivados relatada pelo grupo apresentou-se mais uniforme, e a média foi próxima à recomendada pelo guia.

O grupo de doces e o de bebidas adoçadas também apresentou médias de consumo mais próximas à recomendação do guia ao final da intervenção psicoeducativa. O guia alimentar orienta que o consumo de alimentos do grupo dos açúcares, aí incluídos doces e bebidas processadas com alta concentração de açúcar, seja restrito a uma porção diária, no máximo. Os valores iniciais de ingestão de doces relatados no QFA variaram bastante, ficando a média bem acima do que é recomendado no guia, e o valor máximo apresentado denotou um consumo exacerbado de preparações contendo açúcares simples (19 porções).

O QFA final obteve valor máximo de consumo de doces diário (4 porções) ainda acima do que é recomendado, porém observou-se redução importante quando comparado ao inicial e a média apresentada no final ficou próxima à da orientação do guia. Os valores relativos à ingestão de bebidas adoçadas também apresentaram redução quando comparados os questionários inicial e final aplicados e, nesse grupo, atingiu-se uma média final de consumo que atende às recomendações do guia.

O grupo das preparações incluiu produtos industrializados (molhos de maionese, catchup, mostarda e de tomate), açúcar de adição, extrato de soja e sopas. Não há uma recomendação única no guia que abrange todos esses itens. Porém, sabe-se que os produtos industrializados apresentam comumente elevados índices de sal, bem como podem apresentar altas concentrações de gorduras. No guia alimentar recomenda-se o consumo máximo diário de sal de $5 \mathrm{~g} /$ dia (uma colher de chá rasa) e a redução do consumo de alimentos processados com alta concentração de sal, como temperos prontos, caldos concentrados, molhos prontos, salgadinhos, sopas industrializadas e outros. Recomenda-se também a ingestão de no máximo uma porção diária de alimentos do grupo dos óleos e gorduras, dando preferência aos óleos vegetais, azeite e margarinas livres de ácidos graxos trans. Observou-se redução na ingestão do grupo das preparações; embora não se saiba em qual grupo especificamente foi diminuído o consumo (açúcares, gorduras e sal), foi benéfica a redução, tendo em vista que a diminuição no consumo de preparações contendo esses itens é recomendada pelo guia e é medida de prevenção do desenvolvimento de doenças como obesidade, hipertensão arterial, diabetes e doenças do coração. Resultados semelhantes na avaliação da variação do consumo alimentar em intervenções realizadas com grupos de pacientes com excesso de peso foram encontrados por Alvarez e Zanella (2009), Schneider, Veras, Nascimento e Liberal (2007) sendo que os dois estudos verificaram uma redução no consumo de açúcares e uma redução no peso médio dos participantes.

Diante do que foi mostrado, depreende-se que, embora os resultados do teste do QFA não tenham apresentado valores significativos, os números observados no QFA final, tanto das médias quanto dos valores máximos de ingestão podem sugerir mudança no padrão alimentar, uma vez que tais valores aproximaram-se daqueles índices recomendados no guia alimentar, ou sofreram redução importante. Dessa forma, valores exorbitantes de consumo identificados no QFA inicial foram substituídos por valores normais, compreendidos nas recomendações, que representam um consumo saudável.

\section{Considerações Finais}

Os resultados deste estudo corroboram a literatura ao apontar os benefícios originados pela intervenção grupal e multiprofissional no emagrecimento e estado físico e psíquico do paciente. Constatou-se nesta pesquisa que (1) sentimentos 
como tristeza, baixa autoestima, depressão, ansiedade, motivação e alegria interferem de forma positiva ou negativa na mudança de comportamento alimentar; (2) estratégias de intervenção/ distração, como a prática de atividades físicas, auxiliam na redução de doenças físicas e sintomas psicológicos; e (3) uma ação multiprofissional, por meio de grupos psicoeducativos, que visem à modificação do estilo de vida, é responsável por estimular o paciente a participar do tratamento proposto, obtendo-se respostas terapêuticas mais eficazes. Entretanto, estes dados não são generalizáveis a toda população.

Dentre as limitações deste trabalho estão o reduzido número de participantes avaliados, a ausência de um delineamento experimental com grupo controle e a avaliação bioquímica dos participantes, que não foi possível ser realizada. Possivelmente, a avaliação bioquímica mostraria outros resultados positivos do grupo psicoeducativo que não puderam ser mostrados neste estudo. Sugere-se que futuras pesquisas incluam instrumentos validados que avaliem preditores psicológicos na abordagem em grupo, para se obter uma compreensão mais fidedigna das contribuições da psicologia na mudança do comportamento alimentar. Em suma, espera-se que as reflexões apresentadas neste estudo contribuam para estimular pesquisas sobre o tema e promovam novas estratégias preventivas com foco na saúde e qualidade de vida da população.

\section{Referências}

Alvarez, T. S., \& Zanella, M. T.(2009). Impacto de dois programas de educação nutricional sobre o risco cardiovascular em pacientes hipertensos e com excesso de peso. Revista de Nutrição (Campinas), 22, 71-79.

Anjos, L. A. (1992). Índice de massa corporal (massa corporal.estatura ${ }^{-2}$ ) como indicador do estado nutricional de adultos: revisão da literatura. Revista Saúde Pública, 26, 431-436.

Assis, M. A., \& Nahas, M. V (1999). Aspectos motivacionais em programas de mudança de comportamento alimentar. Revista de Nutrição (Campinas), $12,33-41$.

Associação Brasileira para o Estudo da Obesidade e da Síndrome Metabólica. Consenso latino americano sobre obesidade - ABESO. (1998). Recuperado de http://www.abeso.org.br/pdf/consenso.pdf.

Bardin, L.(2007). Análise de conteúdo. Lisboa: Edições 70.

Beck, J. S. (2009). Pense magro: a dieta definitiva de Beck. Porto Alegre: Artmed

Boza, J., Rech, L., Sachett, L., Menegon, D., \& Cestari, T. (2010). Manifestações dermatológicas da obesidade. Revista HCPA, 30, 55-62.

Cade, M.V., Borloti E., Perin R., Silveira K.A., Ferraz L., \& Bergamim M. P. (2009). Efeitos de um treinamento em autocontrole aplicado em obesos. Cogitare Enfermagem, 14, 65-72.

Cezaretto, A. (2010). Intervenção interdisciplinar para a prevenção de diabetes mellitus sob a perspectiva da psicologia: beneficios na qualidade de vida. (Dissertação de Mestrado não publicada). Universidade de São Paulo, São Paulo.

Chapman, K. M., Ham, J. O., Liesen P., \& Winter L. (1995). Appeying behavioral models to dietary education of eldery diabetic patients. Journal of Nutrition Education, 27, 75-79.

DiMatteo, M. R. (1994). Enhancing patient adherence to medical recommendations. Journal of the American Medical Association, 271, 79-83.

Fonseca, M. J. M., Faerstein, E., Chor, D., \& Lopes, C. S. (2004). Validade de peso e estatura informados e índice de massa corporal: estudo pró-saúde. Revista Saúde Pública, 38, 392-398.

Furlan-Viebig, R., \& Pastor-Valero M. (2004). Desenvolvimento de um questionário de frequência alimentar para o estudo de dieta e doenças nãotransmissíveis. Saúde Pública, 38, 581-584.

Gatti, B.A. (2005). Grupo focal na pesquisa em ciências sociais e humanas. Brasília: Líber Livro.

Instituto Nacional de Câncer - INCA. (2004). Inquérito domiciliar sobre comportamentos de risco e morbidade referida de doenças e agravos não transmissíveis. Rio de Janeiro: Ministério da Saúde.

Instituto Brasileiro de Geografia e Estatística. Pesquisa de orçamentos familiares (2004). Rio de Janeiro. Recuperado de http://www.ibge.gov.br/home/ estatistica/populacao/condicaodevida/pof/2002analise/analise.pdf.

Katz, S., Feigenbaum, A., Pasternary, S., \& Vinker, S. (2005). An interactive course to enhance self efficacy of family practitioners to treat obesity. $B M C$ Medical educative, 5, 4 -11.

Knapp, P., Luz, J. E., \& Baldisserotto, V. (2001). Terapia cognitiva no tratamento da dependência química. In B. Rangé (Org.), Psicoterapias cognitivocomportamentais: um diálogo com a psiquiatria (p. 332-350). Porto Alegre: Artmed.

Krueger, R. A. (1988). Focus group: a practical guide for applied research. Newbury Park, Sage.

Lawrence V. J., \& Kopelman P. G. (2004). Medical consequences of obesity. Clinical Dermatolology, 22, 296-302.

Ministério da Saúde (2008) Guia alimentar para a população brasileira: promovendo a alimentação saudável. Secretaria de Atenção à Saúde. Departamento de Atenção Básica. Brasília, DF.

Monteiro, R. C., Riether, P., Trapp, A., \& Burini, R.C., (2004). Efeito de um programa misto de intervenção nutricional e exercício físico sobre a composição corporal e os hábitos alimentares de mulheres obesas em climatério. Revista de Nutrição, 4, 479-489.

Murta, S.G., (2005). Programas de manejo de estresse ocupacional: uma revisão sistemática da literatura. Revista Brasileira de Terapia Comportamental e Cognitiva, 7, 159-177.

Murta, S. G. (2008). A prática grupoterápica sob o enfoque psicoeducativo: uma introdução. In S. G. Murta (Org.), Grupos psicoeducativos: aplicações em múltiplos contextos (pp. 13-23). Goiânia: Porã Cultural.

Organização Mundial de Saúde (2008). Obesity and overweigth. Recuperado de http://www.who.int/mediacentre/factsheets/fs311/en/index.html

Perez, G. H., \& Romano, B. V., (2004). Comportamento alimentar e síndrome metabólica: aspectos psicológicos. Revista da Sociedade de Cardiologia do Estado de São Paulo, 4, 546-547.

Rangé, B. (2001). Psicoterapias cognitivo-comportamentais: um diálogo com a psiquiatria. Porto Alegre: Artmed.

Rasera, F., \& Rocha, R. (2010). Sentidos sobre a prática grupal no contexto de saúde pública. Psicologia em Estudos, 15, 35 -44.

Santos, P. L. (2010). Efeitos de intervenção interdisciplinar em grupo para pessoas com diagnóstico de sobrepeso ou obesidade. (Dissertação de Mestrado não publicada). Universidade de Brasília, Brasília.

Schneider, C., Veras, A., Nascimento, M. B., \& Liberal, A. (2007). Efeitos de um programa de intervenção nutricional sobre a composição corporal e os hábitos alimentares em obesos em SPA. Revista Brasileira de Obesidade, Nutrição e Emagrecimento, 1, 90-101.

Sharovsky, L., Perez, G. H., Romano, B. W., \& Lopes, H. F. (2004). A psicoterapia de grupo em pacientes portadores de síndrome metabólica. Revista da Sociedade de Cardiologia do Estado de São Paulo, 4, 646-651.

Souza, J. M., Castro, M. M., Maia, E. M., Ribeiro, A. N., Almondes, K. M., \& Silva, N. G. (2005). Obesidade e tratamento: desafio comportamental e social. Revista Brasileira de Terapia Cognitiva, 1(1), 59-67.

Tremblay, A., Sheeran, L., \& Aranda, S. (2008). Psychoeducational interventions to alleviate hot flashes: a systematic review. Menopause, 15, 193-202.

Viana V., (2002). Psicologia, saúde e nutrição: contributo para o estudo do comportamento alimentar. Análise Psicológica, 4, 611-624. 
Cristineide Leandro França, mestre em Psicologia Clínica e Cultura pela Universidade de Brasília, é doutoranda em Psicologia Clínica e Cultura pela Universidade de Brasília e Psicóloga Clínica da Universidade de Brasília. Endereço para correspondência: SMAS, Trecho 1, Lote C, Condomínio Living, Park Sul, Bloco N, Apto 903, Guará, Brasília-DF. E-mail: cristineide@unb.br

Marina Biaginni, nutricionista, é mestranda em Ciências da Saúde pela Universidade de Brasília e nutricionista da Secretaria de Saúde do Distrito Federal. E-mail: marinabiaggini@gmail.com

Ana Paula Levindo Mudesto, especialista em Vigilância Sanitária pela Pontifícia Universidade Católica de Goiás, é nutricionista do Ministério da Aeronáutica. E-mail: anapaula.mudesto@gmail.com

Elioenai Dornelles Alves, doutor em Enfermagem pela Universidade Federal de Santa Catarina, é professor Titular do Departamento de Enfermagem da Universidade de Brasília e coordenador do Laboratório de Educação, EAD e Promoção da Saúde, E-mail: elioenai@unb.br 